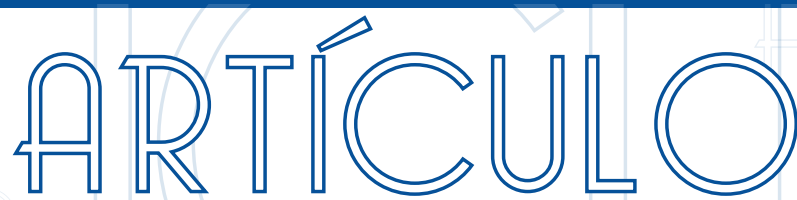

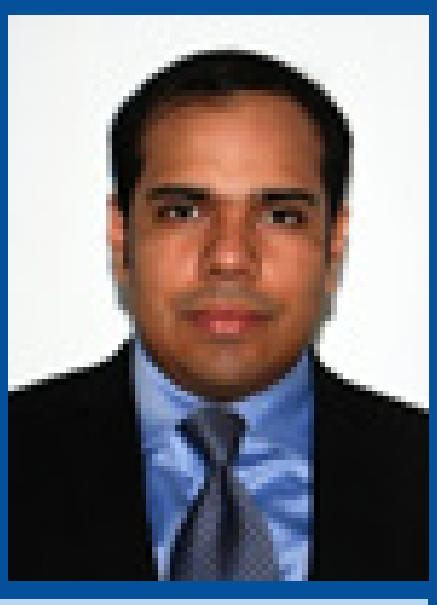

Econ. Ronald Campoverde Aguirre, MEDE.

Economista con Mención en Gestión Empresarial Especialización Finanzas y Magíster en Economía y Dirección de Empresas de la Escuela Superior Politécnica del Litoral. Catedrático de la ESPOL E-mail: rcampoverdea@pucp.pe

CPA. Cecilia Vélez Barros, Mgs. Contadora Pública Autorizada. Magister en Administración de Empresas de la Escuela Superior Politécnica del Litoral. Catedrática de la Universidad de Guayaquil. Catedrática de la Universidad Católica de Santiago de Guayaquil,

E-mail: cecilia.velezba@ug.edu.ec

E-mail: cecilia.velez@cu.ucsg.edu.ec

Ing. Gabriela Aizaga Castro, Mgs.

Ingeniera en Negocios Internacionales. Magíster en Auditoría Integral de la Universidad Técnica Particular de Loja. Analista de Calidad - Universidad Católica de Santiago de Guayaquil.

E-mail: gabrielaaizaga@yahoo.com.

Econ. María José Franco Durán Economista. Analista Empresarial

\section{MODELOS DE INTERNACIONALIZACIÓN DE LOS CANTANTES GUAYAQUILEÑOS}

\author{
MODELS OF INTERNATIONALIZATION OF THE GUAYAQUILEÑOS SINGERS
}

Aceptación: 21/11/2017

Publicación: 05/12/2017

\title{
Resumen
}

El artículo inicia con una descripción general de la globalización musical y la importancia de esta industria para el país. La metodología de investigación seguida fue cualitativa tipo exploratoria. Como herramienta de recolección de datos se utilizó la entrevista semi-estructurada, y la muestra fue escogida de varios artistas internacionalizados o con proyección de internacionalización de diferentes géneros. Se determinaron sus estrategias, proyecciones, motivaciones, factores determinantes y limitaciones, respondiendo a las preguntas de investigación planteadas en este artículo. La investigación busca determinar el modelo de internacionalización utilizado por los cantantes guayaquileños en el contexto actual. Entre los hallazgos más importantes es que modelo de internacionalización varía de acuerdo al género y al capital de inversión disponible, fluctuando entre el modelo evolutivo de la escuela de Uppsala, el modelo trampolín de Pla-Barber y el modelo Born Global de Sigala \& Mirabal. El artículo invita también a explorar el modelo de internacionalización ideal para la industria de la música ecuatoriana.

Palabras clave: internacionalización, distancia psicológica, internalización, productora discográfica, músicos independientes

\section{Abstract}

The paper begins with a general description of the musical globalization and the importance of this industry for the country. The methodology used for the investigation was a qualitative, exploratory type. It was used the semi-structured interviews as a tool for data collection, and the sample was picked from several internationalized artists or with internationalization projections from different music genres. The research aims to determine the internationalization model used by the Guayaquilean singers in the current context. The results determined their strategies, projections, motivations, determinants and constraints, replying to the research questions established in this article. Among the most important findings was that the pattern of internationalization varies according to the music genre and available investment capital, fluctuating between the evolutionary model of the Uppsala school, the trampoline model of Pla-Barber and the Born Global model of Sigala \& Mirabal. The article also invites to explore the ideal internationalization model for the Ecuadorian music industry.

Keywords: internationalization, psychological distance, internalization, record label, independent musicians. 


\section{Introducción}

En las últimas décadas, la industria musical ha pasado por cambios importantes en producción, distribución, comercialización y promoción. Con los desarrollos tecnológicos, producir y distribuir música globalmente es cada vez más fácil. Sin embargo, si no se cuenta con las estrategias adecuadas para una correcta internacionalización, es poco probable que la ventaja competitiva lograda sea suficiente para alcanzar mercados globales.

Varios países han reconocido la importancia de la industria musical para su desarrollo e imagen internacional. Desde hace algunos años, países como España, Uruguay (Ministerio de Educación y Cultura de Uruguay, 2010) y Chile (Gobierno de Chile, 2011) cuentan con programas de apoyo a la internacionalización de la música.

El plan liderado por el Instituto Español de Comercio Exterior (ICEX, 2007), tiene como objetivo desarrollar políticas de promoción internacional en el que participan otras entidades. Los justificativos son (a) la importancia económica de estas industrias; (b) la imagen del país; (c) las oportunidades que ofrecen los mercados extranjeros; $y(d)$ la escasa internacionalización. Tanto Uruguay como Chile han tomado de base el plan español (Ministerio de Educación y Cultura de Uruguay, 2010; Gobierno de Chile, 2011).

Por su parte, el gobierno ecuatoriano actual ha mostrado cierto interés en el apoyo a la música y a la industria de la comunicación, desarrollando incentivos como el Fondo Fonográfico (Ministerio de Cultura y Patrimonio, 2013) y la nueva Ley de Comunicación que intenta proteger la producción nacional (Asamblea Nacional, 2013). Sin embargo, la Revista Líderes (2013), en una de sus publicaciones, sostiene que la poca internacionalización de bandas ecuatorianas se debe al escaso apoyo de las instituciones de gobierno.

En las últimas décadas son pocos los artistas que han alcanzado cierto nivel de internacionalización. El presente estudio plantea, como propósito de investigación, identificar el modelo de internacionalización aplicado por los cantantes guayaquileños en el contexto actual. Las preguntas que se pretenden responder son: (a) ¿Varía el modelo de internacionalización de acuerdo al género musical?; (b) ¿Cuáles son las proyecciones y los factores de internacionalización determinantes para los cantantes guayaquileños?; y (c) ¿Qué limitaciones tienen los cantantes guayaquileños para internacionalizarse?

\section{Marco Teórico}

De acuerdo a Galindo (2009) la "internacionalización se antoja como una herramienta estratégica de desarrollo y consolidación económica del sector".
Ghemawat (2007) destaca que el objetivo central de toda estrategia global, debe ser "gestionar las grandes diferencias que surgen en las fronteras". Por otro lado, Sigala y Mirabal (2013) indican que la globalización ha hecho que los consumidores sean más homogéneos y sus necesidades, preferencias y comportamientos sean menos locales. Esto se debe a las facilidades de distribución a través de los avances tecnológicos y a los acuerdos de integración. Por lo tanto, establecen que se debe tener un enfoque global desde la creación del producto.

Por otra parte, Canals (2004) señala que el crecimiento de una empresa es el medio para lograr los objetivos de la misma, los cuales son satisfacer las necesidades de sus consumidores a través de un producto diferenciado.

En las siguientes líneas se detallan las principales teorías sobre la internacionalización.

\section{Teoría Clásica}

A inicios de 1960, de acuerdo a esta teoría, cada país tenía una dotación fija de mano de obra y capital, y se especializaba en producir una mezcla de productos para obtener el mejor uso posible de sus recursos. Los países con gran número de mano de obra se especializaban en hacer productos que requerían mano de obra intensiva, mientras que los países dotados de gran capital, se especializaban en productos de gran inversión como, por ejemplo, los tecnológicos. Por lo tanto, cada país exportaba su especialidad. Una teoría simple de comercio exterior, en la que el flujo de capital era el mayor incentivo. Sin embargo, con el paso de los años, los países empezaron a notar la brecha de tecnología que manejaban países como Estados Unidos, y el flujo pasó de ser capital a ser un flujo de conocimientos, know-how.

\section{Teoría de Ciclo de Vida del Producto}

El modelo de Vernon (1966) se centra en el análisis de costos de producción y distribución en cada etapa del ciclo de vida del producto. Éste indica que recién en etapa de madurez se puede empezar a considerar una internacionalización del producto, puesto que de esta manera se alcanza una economía de escala y se minimizan riesgos económicos.

\section{Modelo de Uppsala}

Johanson y Vahlne (1977) basan su modelo en la cadena establecida, la distancia psicológica y la teoría de comportamiento de la firma. El modelo plantea que la internacionalización debe ser realizada gradualmente. Araya (2009) señala que el modelo insta a que las empresas inician el proceso de internacionalización cuando todavía son pequeñas, pero se expanden siguiendo los patrones de una estrategia de crecimiento hacia los mercados "psicológicamente" más cercanos. 
Vélez (2013) postula que la falta de conocimiento del mercado extranjero es la mayor barrera de internacionalización, sin embargo la empresa puede superar esto a través de la experiencia. De acuerdo a esto se pueden plantear cinco pasos (a) desarrollar su mercado nacional; (b) iniciar a través de importaciones irregulares; (c) contratar agentes independientes; (d) implantar sucursales comerciales; y (e) poner en marcha las sucursales productivas (Araya, 2009).

\section{Paradigma de Porter}

De acuerdo con Porter (1990), con la globalización la ventaja competitiva es creada y sostenida a través de un proceso altamente localizado. Las diferencias entre los valores, cultura, estructuras económicas e instituciones nacionales contribuyen al éxito competitivo. Sin embargo, las compañías alcanzan una ventaja competitiva a través de la innovación. La innovación se puede manifestar en una característica nueva de un producto, un nuevo proceso de producción, una nueva herramienta de marketing o una nueva forma de dar entrenamiento. Para lograr esta ventaja competitiva, se debe analizar el diamante de atributos detallado en la Figura 1.

\section{Figura 1. Diamante de Ventaja Competitiva}

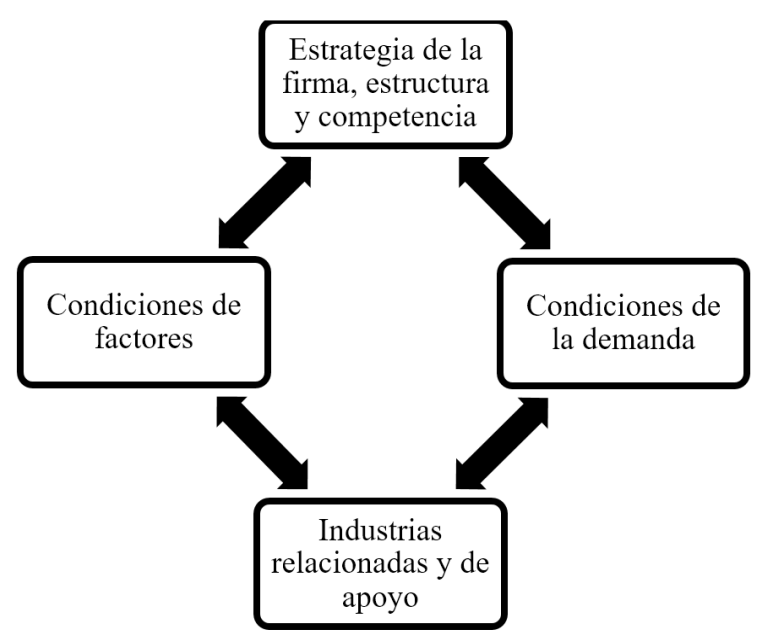

Fuente: Información basado en Porter, M. (1990). The Competitiveness of Nations. New York: Free Press.

\section{Teoría Estratégica.}

El trabajo de Ghoshal se centra en cómo las compañías multinacionales pueden competir más exitosamente y explotar sus ventajas potenciales a las que tienen acceso, en especial esas ventajas que pueden emerger de ubicaciones geográficas dispares y espacios tecnológicos (Portugal, 2011).

A lo largo de sus investigaciones, Barlett y Ghoshal, determinaron que uno de los factores de crecimiento global es implementar una estructura que facilite el flujo de información entre las sucursales con un grado bajo de intervención de la matriz, para que sean interdependientes en un nivel básico.

\section{Teoría de la Internacionalización}

Buckley y Casson (1976) implantaron la internalización en las empresas multinacionales, y determinaron "como uno de los factores importantes para la decisión de gerencia y dirección en la organización de los mercados locales a partir de la Economía de Costes de Transacción".

\section{Paradigma de Dunning}

Esta teoría intenta explicar la inversión extranjera directa de empresas multinacionales y la contribución a las economías locales a través del empleo, tecnología y técnicas de gerencia. Dunning (1988) explica la extensión, la forma y el patrón de la producción internacional, fundamentada en la relación de las ventajas únicas de la empresa, la preferencia de los mercados exteriores y el atractivo de estos mercados para producir ahí, basándose en tres grupos de ventajas. Las ventajas de propiedad son específicas de la empresa, se concentran en la acumulación de activos creados, características tecnológicas y del producto. Las ventajas de internalización coordinan la cadena de valor. Las ventajas de localización son los factores productivos e institucionales de la zona geográfica determinada.

\section{Born Global}

También existe el modelo conocido como Born Global (Sigala \& Mirabal, 2013) o International New Ventures (Pla \& Cobos, 2002) que sigue un proceso de internacionalización mucho más rápido y pasa por encima de un factor de peso en las teorías detalladas anteriormente: la experiencia. Este modelo determina que la estrategia es que las empresas nacen internacionalizadas o se internacionalizan en un corto período de tiempo, dentro de sus primeros años de vida (Madsen \& Servais, 1997).

\section{Trampolín}

La teoría más reciente de internacionalización es el denominado Trampolín. El país trampolín "es una nueva forma de adquirir parte del conocimiento experimental" para entrar a un "nuevo mercado, sin la necesidad inicial de invertir en este": un atajo (Pla-Barber, 2012). El país trampolín debe tener las siguientes características: (a) En relación con el conocimiento institucional, debe tener una posición intermedia entre la oferta y el mercado objetivo, de manera que lo aprendido en el país trampolín pueda trasladarse al país objetivo; y (b) Debe poseer una actividad comercial intensa con el país objetivo, para desarrollar redes desde el país trampolín.

\section{Estrategias de Internacionalización}

Para internacionalizar un producto o empresa se deben responder las siguientes preguntas: ¿Por qué? ¿Cuál es el proce- 
so? ¿Cómo? y ¿Dónde? (Galán, Galende, \& González, 2000). Sin embargo, una sola pregunta puede condicionar todo: ¿qué ventaja competitiva se pretende explotar? (Universidad de Navarra, 2013).

No existe un camino único para la internacionalización, pero de acuerdo a Araya (2009) "existe una misma senda" con opciones en función del control de la empresa sobre su actividad exterior, el riesgo que asume y los recursos que emplea. De acuerdo con Ghemawat (2007), existen tres estrategias genéricas de creación de valor internacional: adaptación, agregación y arbitraje.

Tabla \# 1. Principales teorías sobre la internacionalización

\begin{tabular}{|c|c|c|}
\hline Escuela & Aportación & Autores \\
\hline Teoria Clásica & $\begin{array}{l}\text { Valor de las ventajas } \\
\text { comparativas entre paises }\end{array}$ & Ohlin, 1933 \\
\hline $\begin{array}{l}\text { Teoria del Ciclo de Vida del } \\
\text { Productor }\end{array}$ & $\begin{array}{l}\text { Estudio de la interdependencia } \\
\text { de la vida del producto y la } \\
\text { internacionalización }\end{array}$ & Vernon, 1966 \\
\hline Teoria de Uppsala & $\begin{array}{l}\text { Proceso de } \\
\text { internacionalización de las } \\
\text { empresas }\end{array}$ & $\begin{array}{l}\text { Johanson \& Wiedersheim- } \\
\text { Paul, The intemationalization } \\
\text { of the firm: four Swedish } \\
\text { cases, 1975; Johanson \& } \\
\text { Vahlne, 1977; Johanson \& } \\
\text { Vahlne, 1990; Ohlson \& } \\
\text { Wiedersheim-Paul, } 1978\end{array}$ \\
\hline Paradigma de Porter & $\begin{array}{l}\text { Comparación de las ventajas } \\
\text { de localización en el pais de } \\
\text { origen }\end{array}$ & Porter, 1990 \\
\hline Teoria Estratégica & $\begin{array}{l}\text { Estudio de los requerimientos } \\
\text { estratégicos y organizativos de } \\
\text { la internacionalización }\end{array}$ & $\begin{array}{l}\text { Barlett \& Ghoshal, Managing } \\
\text { accross borders: new strategic } \\
\text { requirements, 1987a; B arlett } \\
\text { \& Ghoshal, 1987b; Barlett \& } \\
\text { Ghoshal, 1989; Hamme1 \& } \\
\text { Pahalad, 1985; Doz, 1986 }\end{array}$ \\
\hline Teoria de Internacionalización & $\begin{array}{l}\text { Enfoque de los costes de } \\
\text { transacción al modo de } \\
\text { internacionalización de la } \\
\text { empresa. }\end{array}$ & $\begin{array}{l}\text { Buckley \& Casson, The } \\
\text { Future of Multinational } \\
\text { Enterprise, 1976; Buckley P. } \\
\text { J., 1988; Hennart, A Theory of } \\
\text { Multinational Enterprise, } \\
\text { 1982; Hennart, 1989; Teece, } \\
\text { 1986; Rugman, Inside the } \\
\text { Multinationals: The } \\
\text { Economics of Internal } \\
\text { Markets, 1981; Rugman, } 1986\end{array}$ \\
\hline Paradigma de Dunning & $\begin{array}{l}\text { Clasificación de los factores } \\
\text { de intemacionalización en } \\
\text { ventajas de propiedad, de } \\
\text { internalización y de } \\
\text { localización }\end{array}$ & $\begin{array}{l}\text { Dunning J. H., Explaining } \\
\text { Changing Patterns of } \\
\text { International Production: In } \\
\text { Defense of the Eclectic } \\
\text { Theory, 1979; Dunning J. H., } \\
\text { 1980; Dunning J. H., 1981; } \\
\text { Dunning J. H., 1985; Dunning } \\
\text { J. H., 1995; Dunning J. H., } \\
1997\end{array}$ \\
\hline
\end{tabular}

Fuente: Información encontrada en Galán, J., Galende, J., \& González, J. (2000). Factores determinantes del proceso de internacionalización: El caso de Castilla y León comparado con la evidencia española. Economía Industrial, 333.

\section{Marco Metodológico}

\section{Diseño de la investigación}

Para el presente estudio se analiza la internacionalización de los cantantes guayaquileños, para lo cual se realizó una investigación cualitativa tipo exploratoria, puesto que el tema no ha sido abordado anteriormente (Creswell, 2013). Se utilizó como herramienta de recolección de datos el método documental y el método descriptivo.

El método documental consiste en un análisis de información existente, escrita o grabada, cuyo objetivo es "establecer relaciones y diferencias" respecto al tema de estudio (Bernal, 2010). Las fuentes de información fueron documentos escritos (revistas científicas, libros, periódicos y bases académicas electrónicas).

Además, se realizaron entrevistas a cantantes guayaquileños para evaluar sus experiencias. Este tipo de investigación está catalogado dentro del método descriptivo. El tipo de entrevista aplicado en esta investigación es la semi-estructurada (APÉNDICE C y APÉNDICE D), pues se requirió flexibilidad en el formato y orden de acuerdo a cada entrevistado (Bernal, 2010). Dado que el propósito de la investigación fue identificar el modelo de internacionalización sin una aplicación contigua, "los resultados son puros o básicos" (Salomón, 2010). Cada entrevista fue grabada y se utilizó como herramienta de apoyo las notas de campo.

El diseño sigue la lógica inductiva, pues parte de lo particular a lo general, permitiendo una comparación entre los datos obtenidos en cada entrevista con la finalidad de lograr una propuesta general para, en cierto grado, pronosticar comportamientos sin generalizaciones cuantitativas (Vieytes, 2004). El horizonte de tiempo para la presente investigación es transaccional, pues el análisis es en un momento único (Hernández, Fernández, \& Baptista, 2003).

\section{Limitaciones}

El presente estudio está sujeto a las siguientes limitaciones (a) la recolección de datos se basa en entrevistas semi-estructuradas realizadas de acuerdo a la disponibilidad de tiempo de los entrevistados (O`Regan \& Ghobadian, 2004); (b) la falta de base de datos para determinar el número de cantantes internacionalizados de Guayaquil es una dificultad para establecer la muestra representativa, por tanto se utilizó un muestreo por conveniencia, lo que disminuye la posibilidad de generalizar los resultados; y (c) no se logró obtener información secundaria de fuentes académicas de prestigio.

\section{Delimitaciones}

Las delimitaciones de la investigación son: (a) la aplicabilidad de los resultados se limita a las estrategias de internacionalización de los cantantes guayaquileños indicados; (b) la investigación fue realizada en Guayaquil; y (c) la unidad de análisis es los cantantes guayaquileños seleccionados que se han internacionalizado o con proyección de internacionalización.

Los datos recogidos en las entrevistas semi-estructuradas fueron analizados siguiendo las técnicas de análisis de discurso (Sounders, Lewis, \& Thornhill, 2009). 


\section{Idoneidad del diseño}

El trabajo de investigación parte de la pregunta cómo, la cual es la base de los estudios descriptivos. Asimismo, supone que el investigador no tiene control total sobre los eventos, pues son fenómenos actuales (Salomón, 2010).

\section{Población y muestra}

Actualmente, Ecuador no cuenta con estadísticas abaladas por una institución formal sobre los cantantes ecuatorianos internacionalizados o con proyección internacional. Por tanto, se ha utilizado varias fuentes para identificar participantes potenciales tal como sugiere Avolio (2008), como información pública en periódicos, revistas y redes sociales, así como referidos por expertos en la industria para determinar qué cantantes son los de mayor proyección internacional al momento.

La muestra se ha determinado mediante la combinación del método de bola de nieve y conveniencia (Sounders, Lewis, \& Thornhill, 2009). Se seleccionaron 14 artistas (13 cantantes y 1 manager), utilizando los criterios de oportunidad y pertinencia (Vieytes, 2004). Los 14 artistas seleccionados son pertinentes, pues forman parte del grupo de artistas guayaquileños internacionalizados o con proyección internacional, y se establece la oportunidad a través de su consentimiento de formar parte de la investigación.

\section{Consentimiento informado}

Las personas que participaron de esta investigación lo hicieron voluntariamente al conceder la entrevista y se informó de manera verbal y/o escrita el propósito de la recolección de datos.

Se solicitó permiso en cada entrevista para utilizar una grabadora para registrar los datos y se confirmó la confidencialidad de dichas grabaciones, reiterando que se utilizaría netamente la información pertinente al objeto de estudio.

\section{Análisis de la información}

El proceso de analizar la información inició tan pronto se transcribieron los datos de las entrevistas. Luego de tener toda la información en una base de datos en Excel, se siguieron los pasos indicados por Avolio (2008) para el análisis de la información (a) codificación abierta (identificar conceptos); (b) codificación axial (relacionar categorías); y (c) codificación selectiva (integrar la teoría).

Se ha realizado un análisis transversal de los resultados, siguiendo los procedimientos sugeridos por Miles y Huberman (1994) y se han examinado los datos bajo el siguiente formato: (a) se definieron dimensiones en base a las preguntas de investigación, entre esas, los antecedentes, motivaciones y limitaciones; (b) se analizaron los datos en común y sus diferencias para así identificar los patrones y sus relaciones; (c) se compararon casos de par en par para encontrar similitudes y diferencias con el fin de encontrar categorías y conceptos no especificados anteriormente (Avolio, 2008).

\section{Resultados y Discusión}

Luego de la investigación de campo realizada, los resultados obtenidos fueron transcritos de acuerdo a las preguntas de estudio. El reporte se detalla en base al análisis transversal de las respuestas. Como propone Avolio (2008), la información obtenida de las entrevistas no es presentada de forma individual, sino de manera dispersa en todo el análisis, dando respuesta a las preguntas de investigación.

\section{Perfil de los informantes}

Se realizaron 12 entrevistas a cantantes guayaquileños que tengan cierto grado de internacionalización o que al menos se estén proyectando internacionalmente, de los cuales dos pertenecen al género de música urbana, dos pertenecen a bandas de rock, uno pertenece al género electrónico y siete pertenecen al género pop que se subdivide en: pop tropical, pop rock, pop country, andipop y baladas.

También se entrevistó al cantante de la banda más reconocida de Ecuador en los 60, Los Corvets, pues brinda información de los cambios en la industria y permite comparar las estrategias utilizadas antes y ahora. Además, para validar ciertos procesos de internacionalización, se entrevistó al manager de uno de los cantantes que participaron del estudio. En la Tabla 2 se detalla el perfil de los informantes, el cual incluye el género, los años en la industria de la música, el grado de inversión, el grado de internacionalización y el grado de profesionalización. 


\begin{tabular}{|c|c|c|c|c|c|}
\hline Nombre & Género & $\begin{array}{l}\text { Años en la } \\
\text { industria }\end{array}$ & Grado de inversión & $\begin{array}{l}\text { Grado de } \\
\text { internacionalización }\end{array}$ & $\begin{array}{c}\text { Grado de } \\
\text { profesionalización }\end{array}$ \\
\hline DJ Victor & Urbano & 6 & Bajo & En proceso & Empírico \\
\hline Mr. Wilson & Urbano & 15 & Bajo & Avanzado & Empírico \\
\hline Nikki Mackliff & $\begin{array}{l}\text { Pop tropical y } \\
\text { baladas }\end{array}$ & 5 & Alto & Inicio & Profesional \\
\hline Barek & $\begin{array}{l}\text { Bomba del Cho- } \\
\text { ta/Pop tropical }\end{array}$ & 5 & Alto & En proceso & Cursos \\
\hline Norka & Electrónica & 7 & Medio & Inicio & Cursos \\
\hline $\begin{array}{l}\text { Juan Santoro (Cadáver } \\
\text { Exquisito) }\end{array}$ & Pop Rock & 6 & Alto & En proceso & Cursos \\
\hline $\begin{array}{l}\text { Pancho Feraud (Abba- } \\
\text { cook) }\end{array}$ & Rock & 5 & Medio & Inicio & Cursos \\
\hline Mirella Cesa & Andipop & 7 & Alto & En proceso & Cursos \\
\hline Kléber Alejandro & $\begin{array}{l}\text { Pop, baladas y } \\
\text { ballenato }\end{array}$ & 9 & Alto & Inicio & Profesional \\
\hline Corvets & $\begin{array}{l}\text { Rock, Pop tro- } \\
\text { pical }\end{array}$ & 20 & Alto & $\mathrm{N} / \mathrm{A}$ & Cursos \\
\hline Danilo Parra & $\begin{array}{l}\text { Pop, salsa y ba- } \\
\text { ladas }\end{array}$ & 11 & Alto & En proceso & Estudia \\
\hline Nicole Pernigotti & $\begin{array}{l}\text { Pop rock y coun- } \\
\text { try pop }\end{array}$ & 1 & Alto & Inicio & Estudia \\
\hline Jorge Luis del Hierro & Baladas pop & 9 & Alto & Avanzado & Cursos \\
\hline
\end{tabular}

\section{Presentación y discusión de resultados}

Los resultados de la investigación se presentan en base a las preguntas de estudio. La información cualitativa se muestra de forma narrativa y en matrices, mientras que la poca información cuantitativa es presentada en gráficos con una breve explicación.

Los datos fueron analizados desde cuatro perspectivas. Primero, se identificaron los datos en común y las diferencias de los entrevistados como: (a) el género musical; (b) años en la industria; (c) grado de internacionalización; (d) grado de inversión; (e) grado de profesionalización; y (f) medios utilizados para promocionarse. Esto ha permitido reconocer patrones comunes de acuerdo al género musical y al capital de inversión.

Luego, se establecieron los diferentes factores determinantes para internacionalizarse. Además, se analizaron las proyecciones y motivaciones de los cantantes, así como las estrategias que utilizan para llegar a los países objetivos.

Asimismo, se identificó un objetivo común entre los cantantes guayaquileños en base a las limitaciones analizadas: firmar con un sello discográfico, por la falta de inversión y la ausencia de disqueras en el Ecuador.

Por último, se combinaron las perspectivas señaladas ante- riormente y se identificaron los modelos seguidos por los cantantes guayaquileños en el contexto actual siguiendo los lineamientos de la revisión bibliográfica.

\section{Patrones comunes de acuerdo al género y capital de inversión}

Los datos proporcionados revelan que las estrategias de internacionalización y el enfoque varían de acuerdo al género y capital de inversión. El género urbano, por ejemplo, cuenta con un capital bajo de inversión tanto para la producción, como para la promoción. Las estrategias más utilizadas para el mercado local son negociar con los DJs de programas de televisión de variedades y farándula de alto rating para que pasen su música. También, suelen apoyarse en los DJs de discotecas para que pongan sus canciones, de esa manera se dan a conocer en el Ecuador.

Para el mercado internacional, apuestan a las redes sociales como videos de sus canciones cargados en Youtube para que puedan difundirse internacionalmente. Si logran algunos hits en un país determinado, es muy probable que empresarios de ese país contacten al artista para una gira, como fue el caso de Mr. Wilson en Perú. Lamentablemente, es importante tener los contactos en el país al que se pretende llegar, pues, a pesar de que las redes sociales están en boga, se pueden perder muchas oportunidades por no tener un vínculo 
que dé retroalimentación al artista y consiga presentaciones. Por otro lado, en el género pop se identifica una tendencia de mayor inversión de capital, pues cuentan con un grupo de trabajo más grande, tanto en producción como en promoción (Figura 2).

Figura 2. Equipo de trabajo

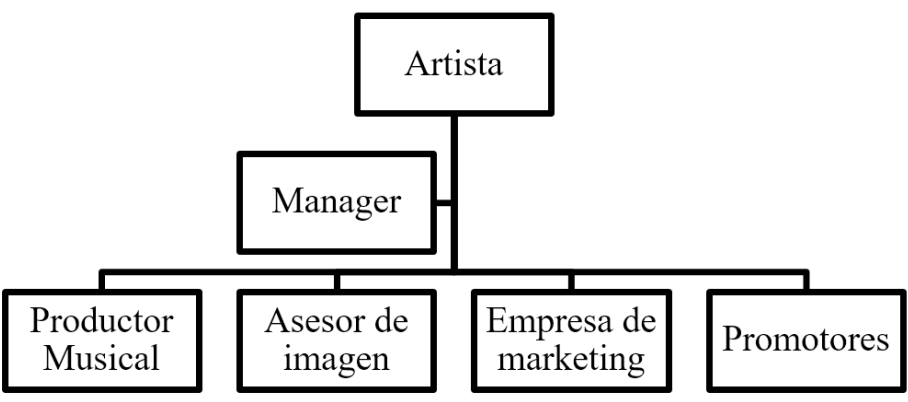

Para la producción musical, a diferencia del género urbano que es mayormente por computadora, utilizan varios instrumentos reales y pasan un proceso de masterización y mezcla, el cual culmina en productoras de otros países, como Estados Unidos o Italia, para dar un mejor acabado.

Además sus actividades promocionales y herramientas utilizadas localmente son de mayor inyección económica, pues se promocionan a través de la televisión, radio, prensa escrita (en algunos casos), y redes sociales. Adicionalmente, cuentan con promotores que "hacen el trabajo hormiga" en el seguimiento de medios como la radio y televisión.

Para el mercado internacional, suelen promocionarse a través de una gira de medios en varios países como es el caso de Mirella Cesa y Nikki Mackliff en Panamá, y Barek en Perú, Costa Rica y Panamá.

En el caso del género electrónico, se entrevistó a la primera expositora de este tipo de música en Ecuador, Norka Cevallos. Sus estrategias de promoción, tanto localmente como internacionalmente, se basa en internet y redes sociales.

Su manager actual, es productor de artistas reconocidos como Paulina Rubio y Gloria Trevi. También indicó que estuvo en Venezuela en el 2009 haciendo gira de medios, país del que fue contactada por una fan, quien consiguió todas las entrevistas. Además, ha viajado a México varias veces auspiciada por un inversionista para realizar presentaciones gratis y negociar un contrato. Sin embargo, no se ha podido llegar a un acuerdo aún.

Por último está el género rock, el cual, al igual que el pop, requiere una inversión alta en producción, puesto que graban con instrumentos reales y para conseguir un sonido de competencia internacional, deben dar los acabados finales en productoras de afuera. Por otra parte, el proceso de promoción está más enfocado en estrategias por redes sociales e internet.

\section{Proyecciones y factores de internacionalización}

El 46\% de los entrevistados indicó que los países a los cuales quieren llegar primero son países donde se escucha su género musical. En el caso del género urbano y del pop tropical, serían países como Perú, Bolivia, Venezuela, Colombia, Panamá, República Dominicana y Costa Rica. El 15\% señaló que el objetivo principal es Estados Unidos, pero para llegar a ese país, se debe llegar primero a países cercanos con una industria musical fuerte como Colombia y México. Sin embargo, el $13 \%$ establece que prefieren concentrarse en el mercado latino. Por otra parte, otro $15 \%$ prefiere concentrarse en mercados lo suficientemente grandes como México y Estados Unidos para hacer carrera ahí.

La Tabla 3 a continuación señala los factores determinantes considerados por los artistas para internacionalizarse.

Tabla \# 3. Factores determinantes para internacionalizarse

\begin{tabular}{|l|c|}
\hline Factor & Porcentaje \\
\hline Calidad & $57 \%$ \\
Contactos & $57 \%$ \\
Disciplina & $50 \%$ \\
Talento & $43 \%$ \\
Una buena canción & $43 \%$ \\
Capital & $36 \%$ \\
Imagen & $21 \%$ \\
Propuesta clara & $21 \%$ \\
Creatividad & $21 \%$ \\
Equipo de trabajo & $21 \%$ \\
Carisma & $14 \%$ \\
Autoconfianza & $7 \%$ \\
Base de fans & $7 \%$ \\
\hline
\end{tabular}

El 70\% considera que consolidarse localmente antes de buscar un mercado internacional es primordial. Las razones presentadas de por qué sí es fundamental consolidarse localmente, fueron básicamente: por referencia, ya que mientras más acogida se tenga en Ecuador, mayores oportunidades de que el cantante sea recibido en otro país. Sin embargo, los que contestaron que no es primordial, sustentaron que es importante, mas no un determinante, mientras se tenga los contactos y herramientas para promover su música en otros mercados.

Los datos recopilados señalan que las motivaciones de internacionalizarse recaen principalmente en consolidarse como artista y mejorar su valoración en la industria. Siete de trece cantantes entrevistados indicaron que uno de sus objetivos de internacionalizarse es crecer económicamente, pues el mercado ecuatoriano no brinda suficientes ingresos para vivir solo de la música. 
En el caso del género urbano, los exponentes manifestaron que la ley seca de los domingos les eliminó un día de trabajo y la reducción de horarios de cierre de las discotecas ocasionaron una baja en el mercado. Además de que, las discotecas en otros países cercanos como Perú y Colombia son mucho más grandes y numerosas, lo que permite al empresario pagarles mejor por show.

Por otro lado, cuatro de los trece cantantes revelaron que desean dejar el nombre de Ecuador en alto. De esta manera, poder posicionar al país como un referente en la industria de la música global para abrir las puertas al resto de artistas nacionales al mercado internacional.

\section{Limitaciones y objetivos en común}

Los resultados evidencian que la mayor fuente de ingreso en la actualidad son los shows. Se puede llegar a tener un buen ingreso por firmar un contrato de imagen, como en el caso de Norka con Movistar. Este ha sido una ventaja importante de la ley de comunicación, donde se incentiva a las empresas a realizar sus publicidades con talento nacional.

En la Tabla 4, se muestran las limitaciones que encuentran los cantantes guayaquileños para internacionalizarse identificadas en el estudio.

Tabla \# 4. Limitaciones para internacionalizarse

\begin{tabular}{|c|c|}
\hline Limitante & Porcentaje \\
\hline Inversión requerida es fuerte & $57 \%$ \\
\hline $\begin{array}{l}\text { Es difícil que el mercado ecuatoriano apoye } \\
\text { el talento nacional }\end{array}$ & $43 \%$ \\
\hline Ausencia de disqueras en el Ecuador & $21 \%$ \\
\hline Falta de identidad cultural & $21 \%$ \\
\hline Falta apoyo de medios & $14 \%$ \\
\hline $\begin{array}{l}\text { El estándar de calidad no es excelente } \\
\text { (competitividad local) }\end{array}$ & $14 \%$ \\
\hline No hay industria musical en el Ecuador & $7 \%$ \\
\hline Falta apoyo de instituciones del gobierno & $7 \%$ \\
\hline $\begin{array}{l}\text { En Ecuador no hay empresas de publicidad } \\
\text { especializadas en artistas }\end{array}$ & $7 \%$ \\
\hline
\end{tabular}

Como se puede observar en la Tabla 4, el principal limitante de los artistas guayaquileños es el capital requerido, por tanto, el objetivo común de los cantantes es lograr firmar con una disquera, en el caso de los cantantes del género urbano y pop, o conseguir un inversionista, en el caso de los cantantes del género rock y electrónica. De esta manera, la tercera parte financia sus carreras, para así implementar una estrategia completa de internacionalización.

Uno de los puntos que ve primero la discográfica es cuántos seguidores tiene el artista en redes sociales. En las Tablas 5 y

6 se detallan los datos de redes sociales de varios cantantes guayaquileños.

Tabla \# 5. Redes sociales

\begin{tabular}{lrrr}
\hline Artista & Facebook & Twitter & Youtube \\
\hline Maykel & 94,359 & 21,600 & $3 ` 597,147$ \\
Rocko y Blasty & 43,160 & 70,300 & $2{ }^{\prime} 634,490$ \\
AU-D & 185,391 & 175,000 & 665,616 \\
Jorge Luis del Hierro & 200,991 & 85,400 & 610,886 \\
Barek & 3,600 & 33,800 & 604,468 \\
L.E.G.O. & 67,389 & 26,700 & 555,780 \\
Daniel Betancourth & 264,912 & 219,000 & 335,620 \\
Juan Carlos Román & 971 & 11,300 & 322,239 \\
Danilo Parra & 98,184 & 43,400 & 209,941 \\
Mirella Cesa & 130,266 & 262,000 & 177,037 \\
Mr. Wilson & 6,052 & 28,100 & 180,427 \\
Danilo Rosero & 331,100 & 128,000 & 171,031 \\
Don Manny & 1,076 & 39,400 & 130,031 \\
Nikki Mackliff & 6,482 & 11,700 & 69,246 \\
Gustavo Herrera & 2,484 & 10,800 & 40,225 \\
Cadáver Exquisito & 15,632 & 5,033 & 38,281 \\
DJ Victor & 72 & 1,743 & 190,971 \\
Norka & 2,712 & 3,441 & 28,361 \\
Kléber Alejandro & 494 & 1,297 & 13,584 \\
ABBACOOK & 943 & 28 & 2,659 \\
\hline Nota: datos constan hasta el 5 de abril de 2014 & &
\end{tabular}


Tabla \# 6

Vistas por día en Youtube

\begin{tabular}{|l|r|}
\hline Artista & Promedio de vistas por día \\
\hline Maykel & $10,248.28$ \\
Rocko y Blasty & $1,610.32$ \\
Daniel Betancourth & 1.498 .30 \\
AU-D & $1,392.50$ \\
Barek & 944.48 \\
Don Manny & 414.11 \\
Mr. Wilson & 343.67 \\
L.E.G.O. & 311.89 \\
Jorge Luis del Hierro & 291.04 \\
Juan Carlos Román & 241.92 \\
Mirella Cesa & 240.87 \\
Cadáver Exquisito & 222.56 \\
Danilo Rosero & 213.26 \\
DJ Victor & 85.52 \\
Danilo Parra & 78.54 \\
Nikki Mackliff & 65.76 \\
Gustavo Herrera & 47.16 \\
Norka & 16.88 \\
Kléber Alejandro & 5.62 \\
ABBACOOK & \\
\hline & \\
&
\end{tabular}

Nota: datos constan hasta el 5 de abril de 2014 
Modelos seguidos por los cantantes guayaquileños en el contexto actual

De acuerdo a la literatura detallada en el marco referencial se pudo determinar que los entrevistados siguen diferentes modelos de internacionalización en base a sus proyecciones y estrategias. El estudio revela que el $67 \%$ de los participantes piensa que el tipo de música es un factor muy importante al momento de internacionalizarse. Además, es un enfoque que se ha venido siguiendo desde Los Corvets, quienes tuvieron que adaptar su música rock a tropical para satisfacer al mercado. Sin embargo el $21 \%$ asegura que un factor determinante es tener una propuesta clara y creatividad, es decir, estar constantemente innovando para ser competitivo.

De acuerdo a Porter (1990) las empresas alcanzan una ventaja competitiva en el mercado global a través de la innovación, pero debe de ser una característica difícil de replicar para que perdure. Por otra parte, el $46 \%$ de los entrevistados sostiene que se proyectan en primera instancia a países cercanos culturalmente, lo que corresponde al Modelo Uppsala, el cual señala que las empresas deben de internacionalizarse gradualmente. Primero desarrollarse dentro de su país de origen y luego expandirse a países psicológicamente cercanos.

Otros sin embargo, aseguran que para llegar a Estados Unidos, primero deben llegar a México, pues éste funciona como país trampolín, ya que cuenta con los contactos requeridos para ser visto por el mercado norteamericano; y a su vez la distancia psicológica es menor entre el país origen y México.

Por otro parte, de los 13 entrevistados, 2 han migrado a otros países para desarrollarse musicalmente desde ahí. El grupo Cadáver Exquisito se trasladó a México donde consiguieron firmar contrato con Universal Music Publishing por los derechos de su primer álbum. También, Nicole Pernigotti, quien está recién iniciando su carrera de cantante, se trasladó a Estados Unidos para producir y promocionarse desde ese país. Esto indica que el modelo al que se apegan sus estrategias es el Born Global, pues como su nombre lo indica nacen internacionalmente, o en el caso de Cadáver Exquisito, se internacionalizaron al poco tiempo de su nacimiento.

\section{Conclusiones y Recomendaciones}

Una vez analizada toda la información recopilada, se puede concluir que los cantantes guayaquileños utilizan diferentes modelos de internacionalización, los cuales pueden ser clasificados de acuerdo al género de música.

En el género urbano, las estrategias señalan que el modelo más próximo es el trampolín de Pla-Barber (2012), pues México funciona como país de enlace para llegar a Estados Unidos. Sin embargo, por la falta de capital primero pasan por Perú y luego Colombia antes de llegar a México, puesto que son países más cercanos psicológicamente. Por tanto, en este lapso siguen el modelo de Uppsala.

El género pop por su parte, ubica mercados culturalmente cercanos para promocionarse y llevar su música como Panamá, Colombia, Venezuela, Perú y Costa Rica. Además, consideran muy importante haberse consolidado primero en el país local antes de buscar un mercado internacional. Estos datos muestran que el modelo de Uppsala es el seguido por este género, ya que es un compromiso gradual como determinado por Johanson \& Vahlne (1977).

Por último en el género rock, los entrevistados apuntan a desarrollarse desde mercados donde la industria musical está más consolidada, es decir que siguen el modelo denominado Born Global (Pla-Barber, 2012), ya que parten se internacionalizan desde el inicio o a los pocos años de haberse constituido.

Así también, se identificó que los factores determinantes de internacionalización son calidad, contactos y disciplina. Mientras que los limitantes principales son la fuerte inversión requerida, la falta de apoyo del mercado ecuatoriano al talento nacional, la ausencia de disqueras en el país y la falta de identidad cultural.

La internacionalización de los artistas guayaquileños requiere de estrategias que partan de modelos de internacionalización que se adapten al contexto actual. Por tanto, se recomienda para futuros estudios, profundizar las limitaciones y oportunidades de los cantantes guayaquileños para determinar el modelo de internacionalización ideal para cada género.

\section{Listado de Referencias}

Araya, A. (2009). El Proceso de Internacionalización de Empresas. Tec Empresarial, III(3), 25.

Asamblea Nacional. (25 de Junio de 2013). Recuperado el 2 de Octubre de 2013, de http://www.andes.info.ec/sites/default/files/pdf/ley-organica-comunicacion-publicada-registro-oficial.pdf

Avolio, B. (Abril de 2008). Un estudio exploratorio del perfil de las mujeres empresarias en el Perú. CENTRUM.

Barlett, C., \& Ghoshal, S. (1987a). Managing accross borders: new strategic requirements. Sloan Management Review, 28(4), 7-17.

Barlett, C., \& Ghoshal, S. (1987b). Managing across boarders: new organizational responses. Sloan Management Review, 29(1), 43-53.

Barlett, C., \& Ghoshal, S. (1989). Managing across boarders: The transnational solution. Harvard Business School Press.

Bernal, C. (2010). Metodología de la Investigación (Tercera edición ed.). Colombia: Prentice Hall.

Boxwell, R. J. (1994). Benchmarking para competir con ventaja. España: McGraw Hill.

Buckley, P. J. (1988). The Limits of Explanation: Testing the Internationalization Theory of the Multinational Enterprise. Journal of International Business Studies, 19(2), 181-193.

Buckley, P. J., \& Casson, H. (1976). The Future of Multinational Enterprise. Londres: MacMillan.

Buckley, P., \& Casson, M. (2009). The internalisation theory of the multinational enterprise: A review of the progress of a research agenda after 30 years. Journal of International Business Studies, 1563-1580.

Canals, J. (2004). Crecimiento empresarial: personas y tencología en la nueva economía. Revista Empresa y Humanismo, II(2), 337-370.

Casillas, J. (1998). El nivel de internacionalización de las empresas españolas con las filiales en el exterior. Investigaciones Europeas de Dirección y Economía de la Empresa, IV(2), 91-108.

Creative Commons Atribución. (2008). El negocio de la música. Recuperado el 8 de octubre de 2013, de http://elnegociodelamusica.blogspot. com/2008/01/3-quin-es-que.html 
Creswell, J. (2013). Research design: Qualitative, Quantitative, and Mixed Methods approaches (Forth Edition ed.). Los Angeles, California, USA: SAGE Publications, Inc.

Doz, Y. (1986). Strategic Management in Multinational Companies. Pergamon Press.

Dunning, J. (1988). The Eclectic Paradigm of International Production: A restatement and some possible extensions. Journal of International Business Studies, 19(1).

Dunning, J. H. (1979). Explaining Changing Patterns of International Production: In Defense of the Eclectic Theory. Oxford Bulleting of Economics and Statics, 41, 269-295.

Dunning, J. H. (1980). Toward an Eclectic Theory of International Production: Some Empirical Tests. Journal of International Business Studies, XI, 9-31.

Dunning, J. H. (1981). International Production and The Multinational Enterprise. Londres: George, Allen \& Unwin.

Dunning, J. H. (1985). Multinational Enterprises, Economic Structure and International Competitiveness. New York: John Wiley \& Sons.

Dunning, J. H. (1995). Revisión del paradigma ecléctico en una época de capitalismo de alianzas. Economía Industrial(305), 15-32.

Dunning, J. H. (1997). Alliance Capitalism and Global Business. New York: Routledge.

Fine, C. A., Vardan, R., Pethick, R., \& El-Hout, J. (15 de Enero de 2002). Rapid-Response Capability in Value-Chain Design. MIT Sloan Management Review.

Galán, J., Galende, J., \& González, J. (2000). Factores determinantes del proceso de internacionalización: El caso de castilla y León comparado con la evidencia española. Economía Industrial, 333.

Galindo, F. (27 de Enero de 2009). La internacionalización de la música española. Obtenido de Expansión: http://www.expansion.com/2009/01/26/ empresas/minegocio/1232998913.html

Ghemawat, P. (2007). Redifining Global Strategy. Apuntes de Globalización $y$ Estrategia IESE.

Gobierno de Chile. (21 de Octubre de 2011). Consejo Nacional de la Cultura $y$ las Artes. Obtenido de http://www.cultura.gob.cl/institucionales/consejo-de-la-cultura-anuncia-plan-de-internacionalizacion-de-la-musica-chilenal

Hammel, G., \& Pahalad, C. (1985). Do you really have a global strategy? Harvard Business Review.

Hennart, J. F. (1982). A Theory of Multinational Enterprise. Ann Arbor, MI: University of Michigan Press.

Hennart, J. F. (1989). Can the new forms of investment substitude for the old forms? A transaction cost perspective. Journal of International Business Studies, $X X(2), 211-234$.

Hernández, R., Fernández, C., \& Baptista, P. (2003). Metodología de Investigación (Tercera Edición ed.). México D.F., México: MacGraw Hill Interamericana.

ICEX. (2007). Plan de apoyo a la internacionalización de Industrias Culturales. Madrid: Ministerio de Economía y Competitividad.

Instituto Ecuatoriano de la Propiedad Intelectual. (22 de Agosto de 2013). Propiedad Intelectual. Recuperado el 12 de Octubre de 2013, de http://www. propiedadintelectual.gob.ec/que-son-derechos-de-autor-y-derechos-conexos/

Johanson, J., \& Vahlne, J. (1977). The internationalization process of the firm: A model of knowledge and development and increasing foreign markets commitments. Journal of International Business, 8, 23-32.

Johanson, J., \& Vahlne, J. (1990). The mechanism of internationalization. International Marketing Review, 7(4), 11-24.

Johanson, J., \& Wiedersheim-Paul, F. (1975). The internationalization of the firm: four Swedish cases. Journal of Management Studies, XII(3), 305-322.

Lema, N., \& Price, A. (11 de Enero de 1995). Benchmarking: Performance Improvement Toward Competitive Advantage. Journal of Management in Engineering, 11(1), 28-37.

Madsen, T., \& Servais, P. (1997). The Internationalization of Born Globals: An Evolutionary Process? International Business Review, 6(6).

Miles, M., \& Huberman, M. (1994). Qualitative Data Analysis: An Expanded Sourcebook (Second Edition ed.). Los Angeles, California, USA: SAGE. Ministerio de Cultura y Patrimonio. (2013). Fondo Fonográfico. Obtenido de http://www.culturaypatrimonio.gob.ec/fondo-fonografico/

Ministerio de Educación y Cultura de Uruguay. (2010). Ministerio de Educación y Cultura. Barcelona.

O'Regan, N., \& Ghobadian, A. (2004). The importance of capabilities for strategic direction and performance. Management Decision, 292-312.

Ohlin, B. (1933). Inter-regional and international trade. Harvard University Press.

Ohlson, H., \& Wiedersheim-Paul, F. (1978). Factors affecting the pre-export behavior of non-exporting firms. (J. Leontiades, Ed.) European Research in International Business.

Park, C. W., Jaworski, B. J., \& Maclnnis, D. J. (Octubre de 1986). Strategic Brand Concept-Image Management. Journal of Marketing, 50(4), 135-145. Obtenido de http://www.jstor.org/discover/10.2307/1251291?ui$d=3737912$ \&uid=2134\&uid=374985881\&uid=374985871 \&uid=2むuid=

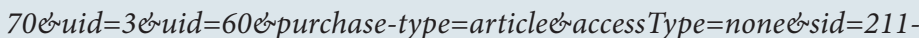
02740678891 \&show MyJstorPss=falseむseq $=1$ \&showAccess $=$ false

Pla, J., \& Cobos, A. (2002). La aceleración del proceso de internalización de la empresa: en el caso de las International New Ventures españolas. Sector Exterior Español(802), 9-22.

Pla-Barber, J. (2012). La filial trampolín. una innovación organizativa en la internacionalización de la emrpesa europea en Latinoamérica. Estudios Gerenciales, 28, 13-25.

Porter, M. (1990). The Competitiveness of Nations. New York: Free Press.

Portugal, M. (2011). Multinationals and subsidiaries: a bibliometric study on Ghoshal's managing accross borders. GlobAdvantage.

Revista Líderes. (4 de Agosto de 2013). Obtenido de www.revistalideres.ec/ informe-semanal/industria_musical-Ecuador-cifras-empleo_0_968903119. html

Rialp, A. (1999). Los enfoques micro-organizativos de la internacionalización de la empresa: una revisión y síntesis de la literatura. ICE(781), 117128.

Rodríguez, M. (2007). Los nuevos desafíos de los derechos de autor en Ecuador. Quito: Abya Yala.

Rugman, A. M. (1981). Inside the Multinationals: The Economics of Internal Markets. New York: Columbia University Press.

Rugman, A. M. (1986). New Theories of Multinational Enterprises: An Assessment of Internalisation Theory. Bulletin of Economics Research, II, 101118.

Salomón, J. (2010). Capacidades Gerenciales en las Empresas Familiares Peruanas: Un estudio descriptivo. CENTRUM.

Sigala, L., \& Mirabal, A. (2013). Empresas Nacidas Globales. Debates IESA, XVIII(2), 54-56.

Sounders, M., Lewis, P., \& Thornhill, A. (2009). Research Methods for Business Students (Fifth Edition ed.). England: Pearson Hall.

Teece, D. J. (1986). Transactions Cost Economics and Multinational Enterprise. Journal of economic Behavior and Organization, VII, 21-45.

Universidad de Navarra. (2013). Internalización Empresarial: Argumentos y estrategias para el directivo. España: Fundación CEDE.

Velez, J. (2013). Internationalization process of a developing country multinational: the outward foreign direct investment decisions in Bimbo Group. Pensamiento \& Gestión(34), 54-68.

Vernon, $R$. (1966). International investment and international trade in the product cycle. Quarterly Journal of Economics, 80, 190-207.

Vieytes, R. (2004). Metodología de la investigación en organizaciones, mercado y sociedad: epistemología y técnicas. Buenos Aires, Argentina: Editorial de las Ciencias. 
Tabla \# 7. Principales países exportadores de música

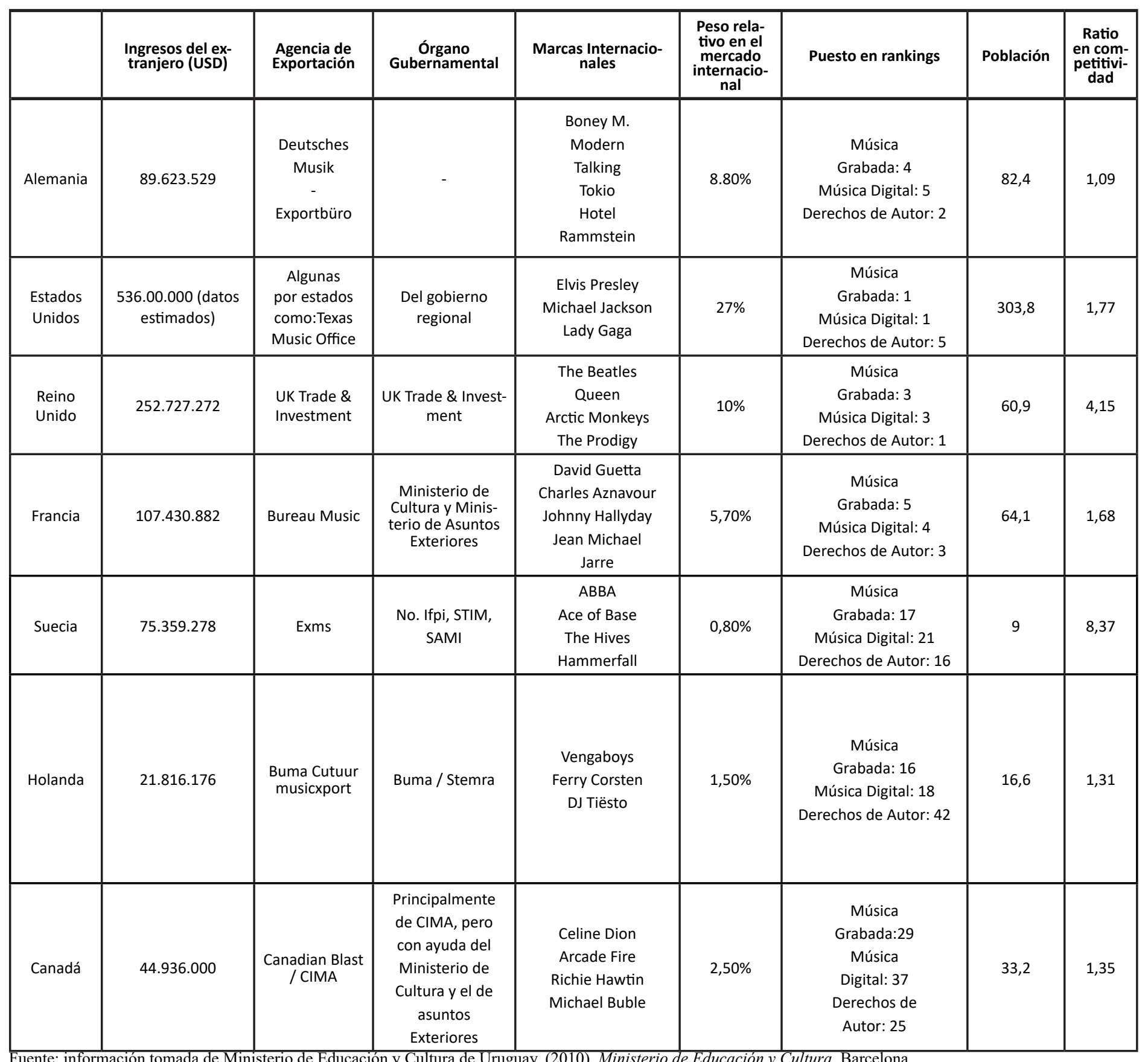

Fuente: información tomada de Ministerio de Educación y Cultura de Uruguay. (2010). Ministerio de Educación y Cultura. Barcelona. 


\section{APÉNDICE B}

Tabla \# 8. El perfil exportador de Argentina y España

\begin{tabular}{|c|c|c|c|c|c|c|c|c|}
\hline & $\begin{array}{l}\text { Ingresos del } \\
\text { extranjero } \\
\text { (USD) }\end{array}$ & $\begin{array}{l}\text { Agencia de } \\
\text { Exporta- } \\
\text { ción }\end{array}$ & $\begin{array}{l}\text { Órgano } \\
\text { Gubernamental }\end{array}$ & $\begin{array}{l}\text { Marcas Interna- } \\
\text { cionales }\end{array}$ & $\begin{array}{l}\text { Peso relativo } \\
\text { en el mercado } \\
\text { internacional }\end{array}$ & $\begin{array}{l}\text { Puesto en } \\
\text { rankings }\end{array}$ & $\begin{array}{l}\text { Pobla- } \\
\text { ción }\end{array}$ & $\begin{array}{l}\text { Ratio en } \\
\text { competiti- } \\
\text { vidad }\end{array}$ \\
\hline 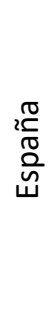 & 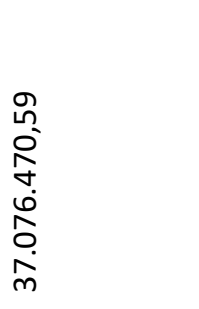 & ICEX / SFS & $\begin{array}{l}\text { Ministerio de In- } \\
\text { dustria; Comercio } \\
\text { y Turismo }\end{array}$ & $\begin{array}{l}\text { Julio Iglesias } \\
\text { Los del Río } \\
\text { Las Ketchup }\end{array}$ & $1,60 \%$ & $\begin{array}{l}\text { Música } \\
\text { Grabada: } 9 \\
\text { Música } \\
\text { Digital: } 11 \\
\text { Derechos de } \\
\text { Autor: } 7\end{array}$ & 45,2 & 0,82 \\
\hline 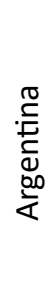 & 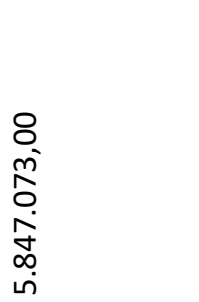 & $\begin{array}{l}\text { OEM (Ar- } \\
\text { gentinean } \\
\text { Music Ex- } \\
\text { port Bureau }\end{array}$ & $\begin{array}{l}\text { Gobierno de la } \\
\text { ciudad de Buenos } \\
\text { Aires }\end{array}$ & $\begin{array}{l}\text { Andrés Calamaro } \\
\text { Mercedes Sosa } \\
\text { Gustavo Santao- } \\
\text { lalla } \\
\text { Astor Piazzolla }\end{array}$ & $0,30 \%$ & $\begin{array}{l}\text { Música } \\
\text { Grabada: } 6 \\
\text { Música } \\
\text { Digital: } 7 \\
\text { Derechos de } \\
\text { Autor: } 11\end{array}$ & 40,5 & 0,14 \\
\hline
\end{tabular}

Fuente: información tomada de Ministerio de Educación y Cultura de Uruguay. (2010). Ministerio de Educación y Cultura. Barcelona

\section{APÉNDICE C.}

\section{Guía de Entrevista a Cantantes}

El presente estudio es realizado para optar el grado de Ingeniera en Gestión de Marketing por la Universidad de Especialidades Espíritu Santo.

El objetivo principal es responder la siguiente pregunta: ¿Cuál es el modelo de internacionalización utilizado por los cantantes guayaquileños en la actualidad?

En este marco, la entrevista pretende identificar, bajo el consentimiento del entrevistado, las estrategias de internacionalización usadas, los resultados obtenidos, los países a los cuáles han llegado, las razones de seleccionar esos países, las limitaciones de ingresar al mercado internacional.

1. ¿Desde qué año incursionó como cantante?

2. ¿Estudió música o aprendió de manera empírica?

3. ¿En qué año incursionó en el mercado internacional?

4. ¿En qué géneros ha incursionado?

5. ¿Qué herramientas utiliza para promocionarse: TV, Radio, Redes Sociales, etc? ¿Por qué?

6. ¿Qué procesos ha pasado su carrera en el ámbito internacional?

7. ¿A qué países ha incursionado y por qué?

8. ¿Qué estrategias ha utilizado para incursionar en esos países?

9. ¿Qué estrategias de internacionalización cree que han dado mayores resultados?

10. ¿Qué resultados ha obtenido en el mercado internacio- nal? ¿cree que existen diferencias entre el consumidor local y el consumidor de otro país?

11. ¿Qué motivaciones tiene para internacionalizarse?

12. ¿Cuáles son sus proyecciones de internacionalización? ¿A qué países quieren llegar y por qué?

13. ¿Qué requiere un cantante guayaquileño para incursionar en el mercado internacional?

14. ¿Qué factores cree que son determinantes para internacionalizarse?

15. ¿Cree que el tipo de música es un factor determinante para internacionalizarse? ¿Extiende, adapta o innova la música de acuerdo al mercado?

16. ¿Cree necesario posicionarse primero en el país de origen antes de buscar un mercado internacional? ¿Por qué?

17. ¿Cuáles cree que son las limitaciones para internacionalizarse? 


\section{APÉNDICE D.}

\section{Guía de Entrevista a Manager}

El presente estudio es realizado para optar el grado de Ingeniera en Gestión de Marketing por la Universidad de Especialidades Espíritu Santo.

El objetivo principal es responder la siguiente pregunta: ¿Cuál es el modelo de internacionalización utilizado por los cantantes guayaquileños en la actualidad?

En este marco, la entrevista pretende identificar, bajo el consentimiento del entrevistado, las estrategias de internacionalización usadas, los resultados obtenidos, los países a los cuáles han llegado, las razones de seleccionar esos países, las limitaciones de ingresar al mercado internacional.

1. ¿Desde qué año incursionó como Manager?

2. ¿Con qué artistas ha trabajado?

3. ¿En qué año incursionó en el mercado internacional?

4. ¿En qué géneros ha incursionado?

5. ¿Qué herramientas utilizan los artistas con los que ha trabajado para promocionarse: TV, Radio, Redes Sociales, etc.? ¿Por qué?

6. ¿Cómo ha sido el proceso de internacionalización de los artistas con los que ha trabajado?

7. ¿A qué países han llegado y por qué?
8. ¿Qué estrategias han utilizado para incursionar en esos países?

9. ¿Qué estrategias de internacionalización cree que han dado mayores resultados?

10. ¿Qué resultados han obtenido en el mercado internacional? ¿cree que existen diferencias entre el consumidor local y el consumidor de otro país?

11. ¿Qué motivaciones tienen para internacionalizarse?

12. ¿Cuáles son las proyecciones de internacionalización? ¿A qué países quieren llegar y por qué?

13. ¿Qué requiere un cantante guayaquileño para incursionar en el mercado internacional?

14. ¿Qué factores cree que son determinantes para internacionalizarse?

15. ¿Cree que el tipo de música es un factor determinante para internacionalizarse? ¿Extiende, adapta o innova la música de acuerdo al mercado?

16. ¿Cree necesario posicionarse primero en el país de origen antes de buscar un mercado internacional? ¿Por qué?

17. ¿Cuáles cree que son las limitaciones para internacionalizarse? 